\title{
Voronoi-Based Archive Sampling for Robust Optimisation
}

\author{
Kevin Doherty \\ K.Doherty@exeter.ac.uk \\ University of Exeter, EX4 4QF, UK \\ Jonathan E. Fieldsend ${ }^{*}$ \\ J.E.Fieldsend@exeter.ac.uk \\ University of Exeter, EX4 4QF, UK
}

\author{
Khulood Alyahya \\ K.Alyahya@exeter.ac.uk \\ University of Exeter, EX4 4QF, UK \\ Ozgur E. Akman \\ O.E.Akman@exeter.ac.uk \\ University of Exeter, EX4 4QF, UK
}

\begin{abstract}
We propose a framework for estimating the quality of solutions in a robust optimisation setting by utilising samples from the search history and using MC sampling to approximate a Voronoi tessellation. This is used to determine a new point in the disturbance neighbourhood of a given solution such that - along with the relevant archived points - they form a well-spread distribution, and is also used to weight the archive points to mitigate any selection bias in the neighbourhood history. Our method performs comparably well with existing frameworks when implemented inside a CMA-ES on 9 test problems collected from the literature in 2 and 10 dimensions.
\end{abstract}

\section{CCS CONCEPTS}

- Theory of computation $\rightarrow$ Evolutionary algorithms; • Computing methodologies $\longrightarrow$ Uncertainty quantification; $\bullet$ Information systems $\rightarrow$ Uncertainty;

\section{KEYWORDS}

Robust optimisation, Voronoi, Fitness approximation, Uncertainty.

\section{ACM Reference Format:}

Kevin Doherty, Khulood Alyahya, Jonathan E. Fieldsend, and Ozgur E. Akman. 2018. Voronoi-Based Archive Sampling for Robust Optimisation. In GECCO '18 Companion: Genetic and Evolutionary Computation Conference Companion, fuly 15-19, 2018, Kyoto, Japan. ACM, New York, NY, USA, 2 pages. https://doi.org/10.1145/3205651.3205768

\section{INTRODUCTION}

The aim in robust optimisation problems is to find a solution that is of high performance quality but which also doesn't incur severe loss of quality when its design variables are disturbed. In this paper, we aim to optimise the expected fitness measure, which we refer to as the effective fitness, $f_{\text {eff }}(\mathbf{x})$. To do so efficiently, we can use previously evaluated designs visited during the search process. However, a bias can be introduced in the distribution of archived solutions and a

\section{${ }^{*}$ Corresponding author}

Permission to make digital or hard copies of all or part of this work for personal or classroom use is granted without fee provided that copies are not made or distributed for profit or commercial advantage and that copies bear this notice and the full citation on the first page. Copyrights for components of this work owned by others than the author(s) must be honored. Abstracting with credit is permitted. To copy otherwise, or republish, to post on servers or to redistribute to lists, requires prior specific permission and/or a fee. Request permissions from permissions@acm.org.

GECCO '18 Companion, Fuly 15-19, 2018, Kyoto, Japan

() 2018 Copyright held by the owner/author(s). Publication rights licensed to Association for Computing Machinery.

ACM ISBN 978-1-4503-5764-7/18/07 . \$15.00

https://doi.org/10.1145/3205651.3205768 number of approaches propose weighting and re-sampling schemes to mitigate this $[2,4]$.

For a given design point, $\mathbf{x}$, we define its uncertainty neighbourhood, $U(\mathbf{x})$, as the volume in design space over which we wish to calculate its effective fitness. We define the extended uncertainty neighbourhood, $U^{*}(\mathbf{x})$, as the volume that lies within 1.5 times the radius of $U(\mathbf{x})$. The Voronoi tesselation of a set of discrete points in a continuous space divides the space into a set of cells so that each point's cell contains the volume of space that is closest to it. It also has the property that the point furthest from all of the generating points will be one of the vertices of the cells. Voronoi-Based Archive Sampling (VBAS) is described in Figure 1. The effect of this method is to perform an integration over $U(\mathbf{x})$, with each element of the uncertainty domain assigned the value of the (Euclidean) closest design point in the archive. In this paper, we present a Monte-Carlo approximation (MC+VBAS) and compare against existing methods in the literature on a set of nine test problems. We demonstrate that $\mathrm{MC}+\mathrm{VBAS}$ performs comparably well to the current state-of-the-art and significantly better on some problems.

\section{EXPERIMENTAL DESIGN}

We ran the following set of experiments:

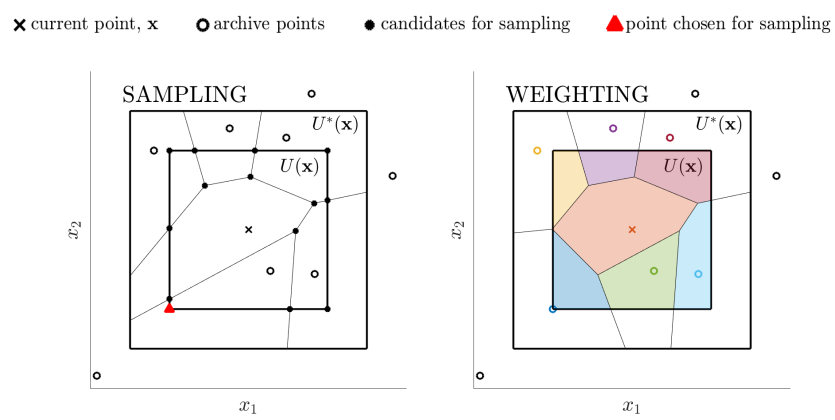

Figure 1: An illustration of the VBAS algorithm for dimension size $d=2$. In the first step (left), a bounded Voronoi diagram is generated within $U^{*}(x)$ and truncated to within $U(\mathrm{x})$. We sample the vertex of the truncated Voronoi diagram that maximises the minimum distance to any of the archive points. Next (right), we update the Voronoi tesselation to include the sampled point. The effective fitness of $x$ is estimated by a weighted sum of the fitnesses of the coloured points. The weights are given by the relative volumes of the intersections of each Voronoi cell with $U(x)$. The colour of each point used for weighting matches the coloured area. 


\begin{tabular}{|l|c|c|c|c|c|c|c|c|c|}
\hline $\mathbf{d}=\mathbf{2}$ & TP1 & TP2 & TP3 & TP4 & TP5 & TP6 & TP7 & TP8 & TP9 \\
\hline OL & $0.58(0.00)$ & $-1.80(0.10)$ & $1.14(0.00)$ & $-0.46(0.00)$ & $-0.64(0.04)$ & $0.33(0.00)$ & $-0.38(0.02)$ & $-0.31(0.00)$ & $0.00(0.06)$ \\
SEM+AR & $0.21(0.00)$ & $-1.78(0.05)$ & $0.89(0.05)$ & $-0.83(0.00)$ & $-0.66(0.01)$ & $0.16(0.01)$ & $-0.40(0.01)$ & $-0.40(0.01)$ & $-0.11(0.02)$ \\
LHS+ASA & $0.21(0.00)$ & $-1.78(0.10)$ & $0.84(0.01)$ & $-0.82(0.01)$ & $-0.67(0.01)$ & $0.15(0.00)$ & $-0.41(0.00)$ & $-0.38(0.01)$ & $-0.11(0.03)$ \\
MC+VBAS & $\mathbf{0 . 2 1}(0.00)$ & $-1.83(0.00)$ & $0.83(0.00)$ & $\underline{-0.83}(0.00)$ & $-0.68(0.00)$ & $\underline{\mathbf{0 . 1 4}}(0.00)$ & $\underline{-0.42}(0.00)$ & $-0.40(0.00)$ & $\underline{-0.13}(0.01)$ \\
min f $_{\text {eff }}$ & $\mathbf{0 . 2 1}$ & $-\mathbf{1 . 8 9}$ & $\mathbf{0 . 8 3}$ & $-\mathbf{0 . 8 3}$ & $-\mathbf{0 . 6 8}$ & $\mathbf{0 . 1 4}$ & $-\mathbf{0 . 4 2}$ & $-\mathbf{0 . 4 0}$ & $-\mathbf{0 . 1 7}$ \\
\hline \hline $\mathbf{d = 1 0}$ & TP1 & TP2 & TP3 & TP4 & TP5 & TP6 & TP7 & TP8 & TP9 \\
\hline OL & $2.91(0.00)$ & $-8.49(0.37)$ & $6.02(0.11)$ & $-0.47(0.01)$ & $-0.63(0.02)$ & $0.77(0.00)$ & $-0.39(0.00)$ & $-0.25(0.02)$ & $-0.02(0.01)$ \\
SEM+AR & $1.36(0.11)$ & $-8.35(0.26)$ & $5.49(0.40)$ & $-0.79(0.02)$ & $-0.63(0.02)$ & $0.16(0.00)$ & $-0.39(0.01)$ & $-0.31(0.02)$ & $-0.05(0.03)$ \\
LHS+ASA & $1.07(0.02)$ & $-8.54(0.25)$ & $5.03(0.30)$ & $-0.82(0.01)$ & $-0.63(0.03)$ & $0.15(0.00)$ & $-0.40(0.00)$ & $-0.34(0.03)$ & $-0.04(0.02)$ \\
MC+VBAS & $1.06(0.01)$ & $-8.51(0.22)$ & $5.04(0.26)$ & $-0.82(0.00)$ & $-0.64(0.02)$ & $\underline{\mathbf{0 . 1 5}(0.00)}$ & $-0.39(0.00)$ & $-0.32(0.02)$ & $-0.03(0.02)$ \\
min f & $\mathbf{1 . 0 4}$ & $-\mathbf{9 . 4 7}$ & $\mathbf{4 . 1 7}$ & $-\mathbf{0 . 8 3}$ & $-\mathbf{0 . 6 8}$ & $\mathbf{0 . 1 4}$ & $-\mathbf{0 . 4 2}$ & $-\mathbf{0 . 4 0}$ & $-\mathbf{0 . 1 7}$ \\
\hline
\end{tabular}

Table 1: Effective fitness of solutions returned by CMA-ES for $d=2$ (top) and 10 (bottom). Each entry shows the median effective fitness over 30 runs with the median absolute deviation in parentheses. Results that are significantly better than all others are in red and underlined. Significance was determined using a signed rank test with significance level 0.05, corrected using the Holm-Bonferroni correction. OL was not included in the statistical testing. Results are rounded to two decimal places.

OL: Optimising on the Original Landscape.

SEM+AR [1]: The fitness of a design is estimated using all designs from the archive in its neighbourhood and one additional random sample. All the designs are weighted equally.

LHS+ASA [2]: A set of reference points in the neighbourhood of a design are generated from a Latin hypercube. An approximation to the Wasserstein distance is used for sampling and weighting.

MC+VBAS: Approximate VBAS, where a number of reference points are generated from a uniform distribution over the disturbance neighbourhood. The one that is farthest from any of the archive points is chosen for sampling and the archive points are weighted by the number of reference points that count them as their nearest neighbour.

We incorporated all of the aforementioned methods into a standard CMA-ES [3], using mostly the default parameters, except that we give equal weights to all children for recombination. If the disturbance neighbourhood extends beyond the boundary, we truncate
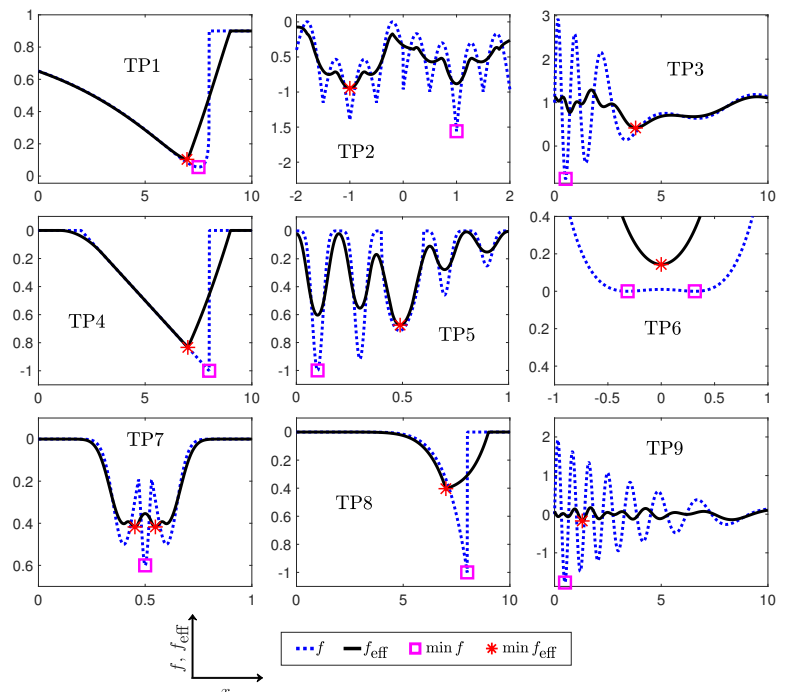

Figure 2: Visualisations of the test functions when $d=1$. The plot of TP6 is zoomed in to highlight the interesting region. it to the boundary. Experiments are executed with fixed budgets of 1000 and 5000 evaluations for dimension size, $d=2$ and 10, respectively. The number of Latin hypercube samples used in LHS+ASA is set to $3^{d}$ for $d=2$ and $3^{5}$ for $d=10$, following the settings used in [2]. ${ }^{1}$ A fixed number of 1000 samples is used in MC+VBAS for each dimension size.

We assess the performance of the methods on nine test problems. TP1-3 are from [2], and TP4-9 from [5] (with disturbance distributions modified to be uniform). Visualisations of the original and robust (effective) landscapes are shown in Figure 2 for $d=1$.

\section{RESULTS \& DISCUSSION}

Table 1 shows the median $f_{\text {eff }}$ achieved by each method on each test problem, for each value of $d$. In the final iteration of CMA$\mathrm{ES}$, the barycenter of the solutions chosen as parents is returned. $M C+V B A S$ performs significantly better than all other methods on some problems. It is notable that the performance of SEM+AR is quite good in a number of cases despite not accounting for the bias in the distribution of archive points in any way. OL performs well on a number of test problems, raising the question of the effectiveness of these for robust optimisation assessment.

\section{ACKNOWLEDGEMENTS}

This work was supported by the Engineering and Physical Sciences Research Council [grant number EP/N017846/1].

\section{REFERENCES}

[1] J. Branke. 1998. Creating robust solutions by means of evolutionary algorithms. In Parallel Problem Solving from Nature - PPSN V: 5th International Conference. 119-128.

[2] J. Branke and X. Fei. 2016. Efficient Sampling When Searching for Robust Solutions. In Parallel Problem Solving from Nature - PPSN XIV: 14th International Conference. 237-246.

[3] N. Hansen and A. Ostermeier. 2001. Completely Derandomized Self-Adaptation in Evolution Strategies. Evolutionary Computation 9, 2 (2001), 159-195.

[4] J. Kruisselbrink, M. Emmerich, and T. Bäck. 2010. An Archive Maintenance Scheme for Finding Robust Solutions. In Parallel Problem Solving from Nature, PPSN XI: 11th International Conference. 214-223.

[5] I. Paenke, J. Branke, and Y. Jin. 2006. Efficient search for robust solutions by means of evolutionary algorithms and fitness approximation. IEEE Transactions on Evolutionary Computation 10, 4 (2006), 405-420.

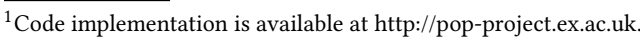

